\title{
The Role of Fins for the Diffusion Limiting Current in a Stacked Rotating Bipolar Electrode Cell
}

\author{
Takeshi SASAKI* and Tatsuo ISHIKAWA
}

Received September 12, 1987 ; Accepted December 10, 1987

\begin{abstract}
The role of fins for mass transfer in a stacked rotating bipolar electrode cell was investigated by measuring the diffusion limiting current under variety of heights and number of fins with $0.01 \mathrm{M}$ ferri/ferrocyanide in a $1 \mathrm{M}$ NaOH aqueous solution ( $M=m o 1 \cdot \mathrm{dm}^{-3}$ ). The distribution of the diffusion limiting current on the electrode surface was also determined using ring electrodes. The rotation of the fins attached to the electrodes flattens the distribution of the limiting current across the electrode surface and simultaneously elevates the mass transfer rate. The distance of $1 \mathrm{~mm}$ from the top of fins to the opposite surface is small enough to raise the effective agitation to the opposite surface. The limiting current is little affected by the variation in the height and the number of fins.
\end{abstract}

\section{Introduction}

A stacked rotating bipolar electrode cell has been developed for the electro1ytic recovery of metal from dilute solutions. $(1,2)$ In the cell, a high speed metal recovery and a high current efficiency are achieved owing to the excellent agitation effect of fins attached to the electrodes, and also owing to the surface micro-turbulence and increased active surface area caused by powdery copper deposition. The characteristics for mass transfer in the cell with four $2 \mathrm{~mm}-\mathrm{high}$ fins has been investigated in three cells of different sizes.( 3 )

In this paper, the role of fins for the mass transfer were studied by measuring the diffusion limiting current under variety of fin configurations and by determining the distribution of mass transfer on the electrode surfaces.

\section{Experimental}

The three test cells (F-21, F-52, F-92, where the numerical values indicate the working areas of one electrode in $\mathrm{cm}^{2}$ ) and the test solution were the same as reported elsewhere.(3) The cell consisting of one rotor and two stator electrodes is schematically shown in Fig. 1. The cell comprises a unit pair of stator and rotor of the stacked rotating bipolar electrode cel1. Two surfaces plated with nickel were investigated: $S-1$, the stator surface opposite to the rotating fins, and $\mathrm{S}-3$, the rotor surface opposite to the stationary fins. The interelectrode gap $(1 \mathrm{~g})$ between the rotor and the stator was varied from 2 to 6 $\mathrm{mm}$, the height of fins $\left(f_{h}\right)$ was 2 or $5 \mathrm{~mm}$, and 4 or 8 fins were attached on the anode surfaces.

Ring electrodes in the F-21 ce11 were used for measuring the 1 ocal diffusion limiting current $\left(j_{L}\right)$ and for determining the distribution of mass transfer on the electrode surfaces. The schematic diagrams and the sizes of the electrodes are shown in Fig. 2, The ring electrode were graphite rods worked into ring shape and covered with epoxy resin, so the ring remained exposed (Fig. 2). The ring-shaped surface was used as a ring electrode after being faced-off on a

Department of Metal1urgical Engineering, Faculty of Engineering, Hokkaido University, Sapporo 060 .

Rey Words: Diffusion Limiting Current, Bipolar Electrode, Rotating Electrode. 


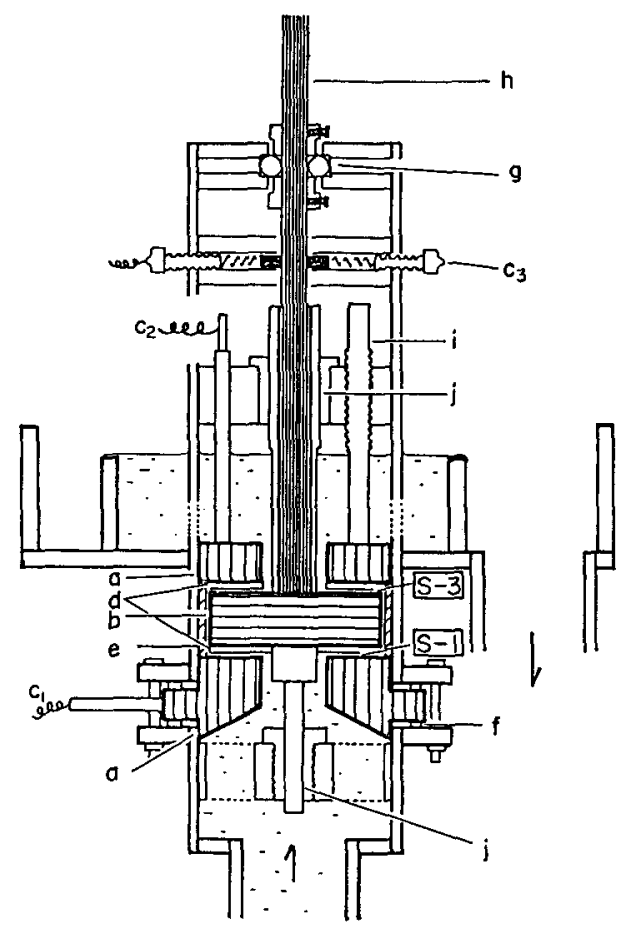

lathe and it was plated with nickel. Four ring electrodes of different radii were prepared for both the $S-1$ and the S-3 surfaces and their sizes are presented in the bottom of the figure. The apparent surface area ranged from 1.2 to $3.2 \mathrm{~cm}^{2}$.

The rotational speed ( $\omega$ ) was varied from 0 to $2000 \mathrm{rpm}$. The volumetric flow rate per unit height of interelectrode gap ( $q$ ) was varied from 0 to $2080 \mathrm{~cm}^{2}$. s-1. The details of the experimental techniques were a 1 so reported.(3)

Fig. 1 Test cel1.

(a) stator, (b) rotor, (c1, c $c_{2}, c_{3}$ ) 1ead wire, (d) acrylic fin, (e) acrylic spacer, (f) silicone rubber packing, (g) bal1 bearing, (h) rotating shaft, (i) fixing rod, and ( $j$ ) pillow block.

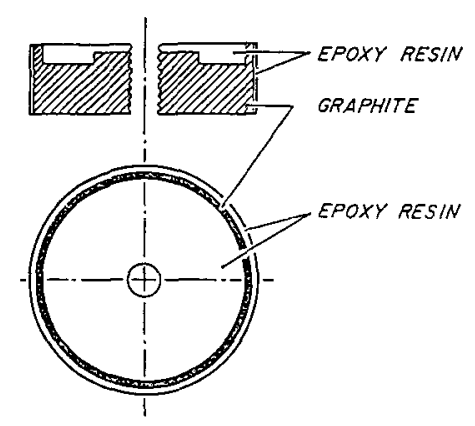

$s-3, d^{\prime}$

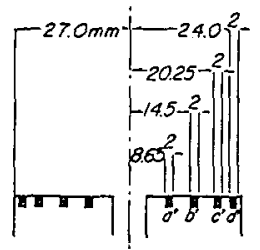

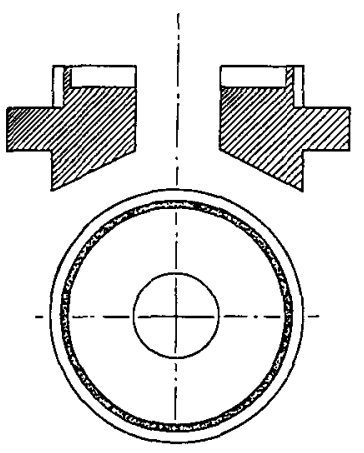

$s-1, d$

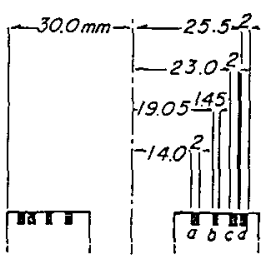

Fig. 2 Schematic diagram of ring electrodes: rotor (S3 , d')-left and stator ( $-1, d)-r i g h t$, and the sizes of ring electrodes; $a, b, c$, and d indicate the location of the rings on the $S-1$ surface, and $a^{\prime}, b^{\prime}, c^{\prime}$, and $d^{\prime}$ indicate that on the $S-3$ surface. 


\section{Results and discussion}

In F-21 without attached fins, the variation of the limiting currents with the volumetric flow rate per unit height at various rotational speeds is shown in Figs. 3 and 4. The flow of electrolyte affected the limiting currents, but the effect of rotational speed is smal1 on both surfaces. The limiting current on the S-1 surface for the rotating electrodes at low flow rates decreases with increases in the volumetric flow rate. A similar change was also observed on the S-3.surface above $700 \mathrm{rpm}$.

Fleischmann et a1. reported(4) that the fluid in the interelectrode gap in a capillary gap cel1 and in a pump cell can be modeled as a three-zone flow, with a nearly stagnant creeping layer close to either electrode and a faster core

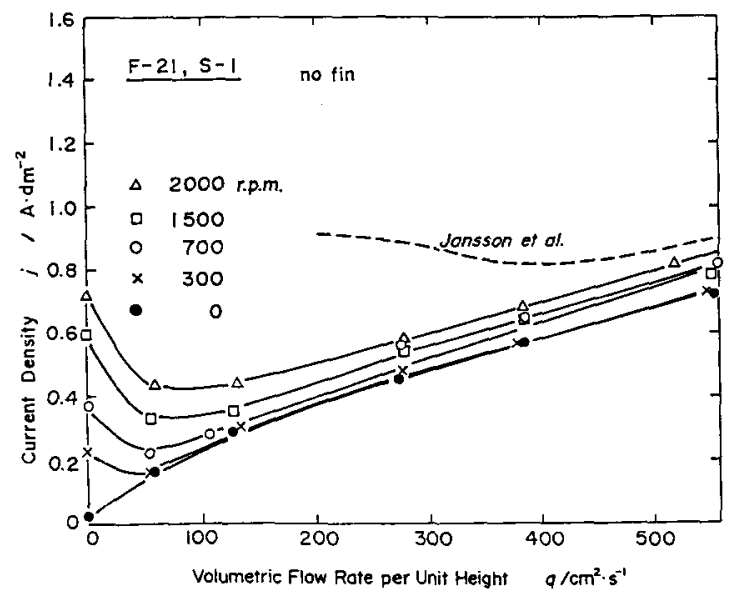

Fig. 3 Diffusion limiting current density as a function of the volumetric flow rate per unit height of interelectrode gap at various rotational speeds on $\mathrm{S}-1$ in F-21 without fins.

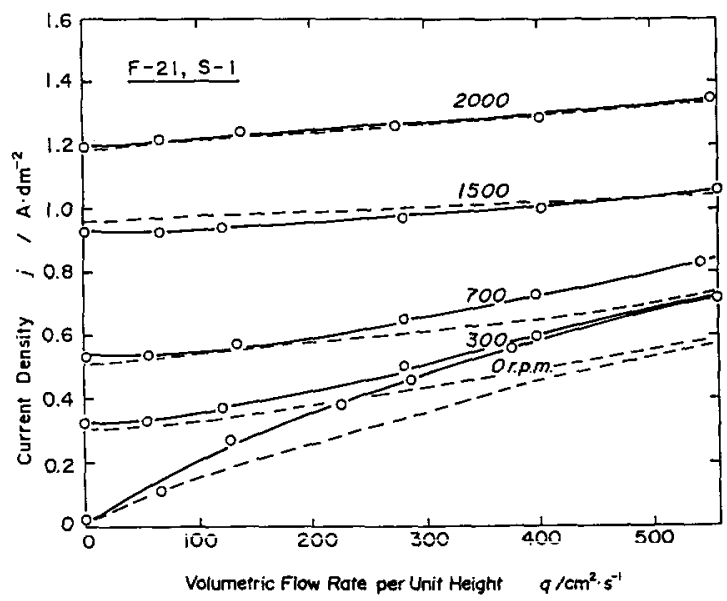

Fig. 5 Diffusion 1 imiting current density as a function of the volumetric flow rate per unit height at various rotational speeds on $\mathrm{S}-1$ in F-2I. Four fins with $\mathrm{f}_{\mathrm{h}}=2 \mathrm{~mm}$.

Solid line: $1_{g}=3 \mathrm{~mm}$; broken line: $1_{g}=2 \mathrm{~mm}$.

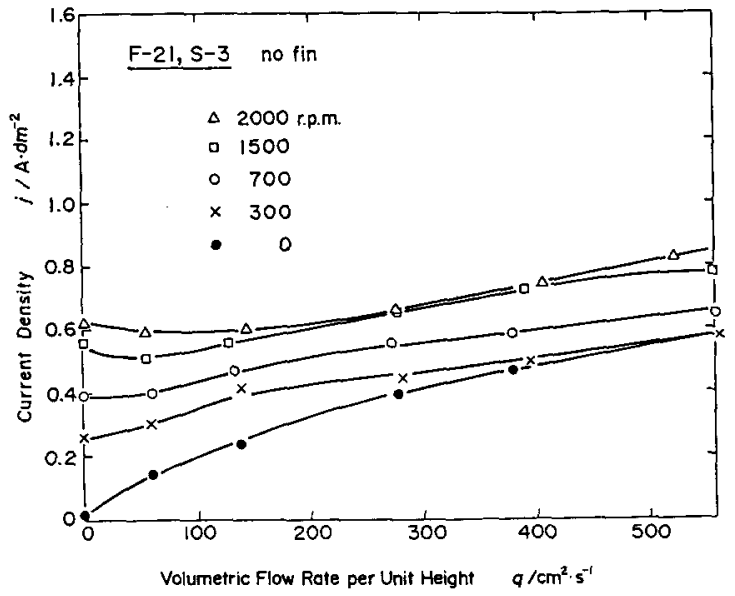

Fig. 4 Diffusion limiting current density as a function of the volumetric flow rate per unit height of interelectrode gap at various rotational speeds on $\mathrm{S}-3$ in $\mathrm{F}-21$ without fins.

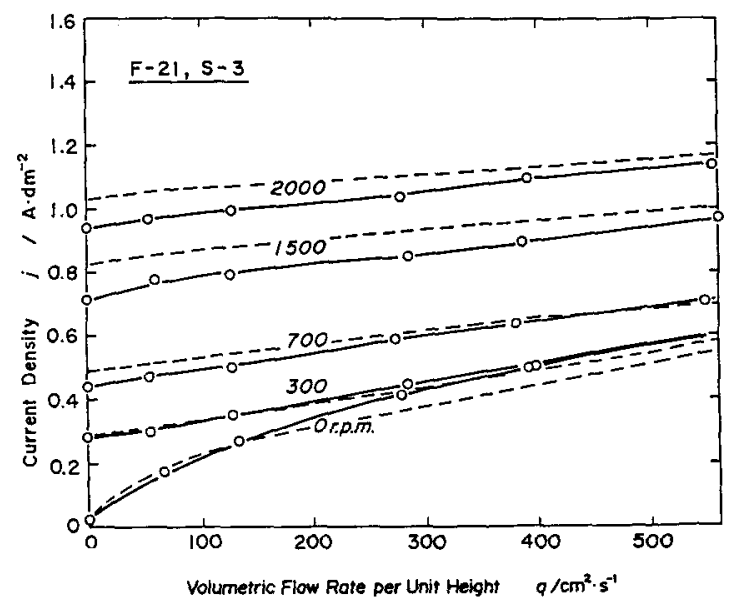

Fig. 6 Diffusion 1imiting current density as a function of the volumetric flow rate per unit height at various rotational speeds on $S-3$ in F-21. Four fins with $f_{h}=2 \mathrm{~mm}$.

Solid 1 ine: $1_{\mathrm{g}}=3 \mathrm{~mm}$, broken 1 ine: ${ }^{1} \mathrm{~g}=2 \mathrm{~mm}$. 
flow in between.

The results from Jansson et al. (2800 rpm, $\left.1_{\mathrm{g}}=0.76 \mathrm{~mm}\right)(5)$ is also shown in Fig. 3. Compared with the present results it shows a very high 1 imiting current density especially at low flow rates. This may be due to the very smal1 interelectrode gap, on 1 y $0.76 \mathrm{~mm}$, where the rotational effect of the electrode easily spreads to the opposite electrode. The small rotation effect in the present results is probably due to the rather large interelectrode gap, $3 \mathrm{~mm}$, since the electrolyte can easily flow through the interelectrode core almost independent of the rotation of the electrodes. It seems that the height of the interelectrode gap is important for the mass transfer in the electrode system without fins. In the absence of electrolyte flow, when there is no core flow between the electrodes, the creeping 1 ayer at the rotor can develop and will cause high turbulence which promotes mass transfer. This leads to the increase in the 1 imiting current at 1 ow flow rates as shown in the figures.

The diffusion 1 imiting currents on the S-1 and S-3 surfaces in the F-21 cel1 having electrodes with fins are shown in Figs. 5 and 6 . The results from $1 \mathrm{~g}=2$ $\mathrm{mm}$, where the fins scrape the opposite surfaces slightly, are also presented in these figures (broken lines). The figures show that the rotation effect on the limiting currents is very high and that the effect of the flow rate becomes small in the high rotational speed range. This is probably caused by an extremely disturbed core flow between the electrodes. Comparing Figs. 5 and 6 with Figs. 3 and 4, the effect of the fins on the 1 imiting currents are remarkable especial1y at high rotational speeds. Figures 5 and 6 also show that differences in interelectrode gaps ( 2 and $3 \mathrm{~mm}$ ) have no significant effect on the 1 imiting current. This suggests that a gap of $1 \mathrm{~mm}$ from the top of fins to the opposite surface is small enough for the agitation effect to transfer to the opposite surface.

The 1 imiting current distribution on S-1 and S-3 in the F-21 cel1 using electrodes with fins is shown in Figs. 7 and 8. The diffusion 1 imiting current could not be determined at the flow rate of $56 \mathrm{~cm}^{2}$. $\mathrm{s}^{-1}$ and $2000 \mathrm{rpm}$ on the S-1 surface because gas induced by the fins stayed around the rotating axis.

Without rotation, the limiting currents on both surfaces which increase with the flow rate decrease from the center to the periphery. This reflects the flow rate distribution between electrodes, since the radial flow of electrolyte decelerates from the center towards the periphery on S-1 and it accelerates from the periphery towards the center on S-3. At $700 \mathrm{rpm}$, there is a discernib1e effect of the flow rate distribution, but it disappears at higher rotational speeds.

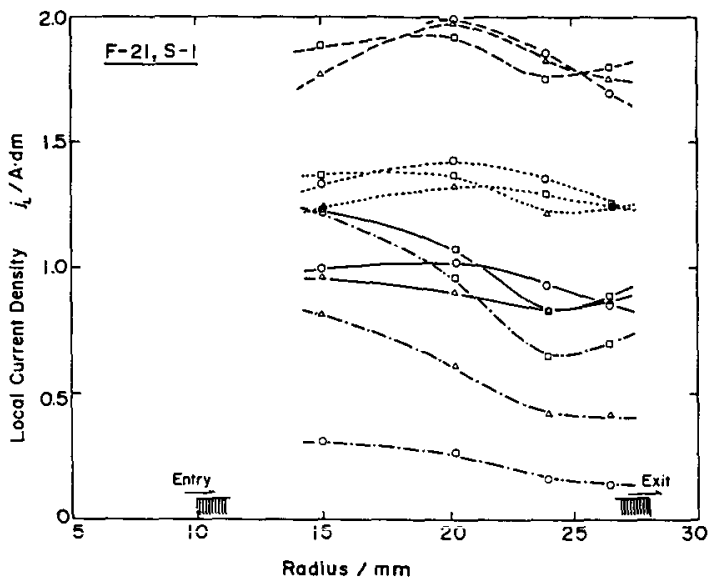

Fig. 7 Distribution of limiting current density on S-1 in F-21. $1 \mathrm{~g}=3 \mathrm{~mm}$. Four fins with $f_{h}=2 \mathrm{~mm}$.

Rotational speed ( $\mathrm{rpm}):--0,-700, \cdots 1200$, --2000 . Volumetric flow rate $\left(\mathrm{cm}^{2} \cdot \mathrm{s}^{-1}\right)$ : $0-56, \Delta-278, \square-556$.

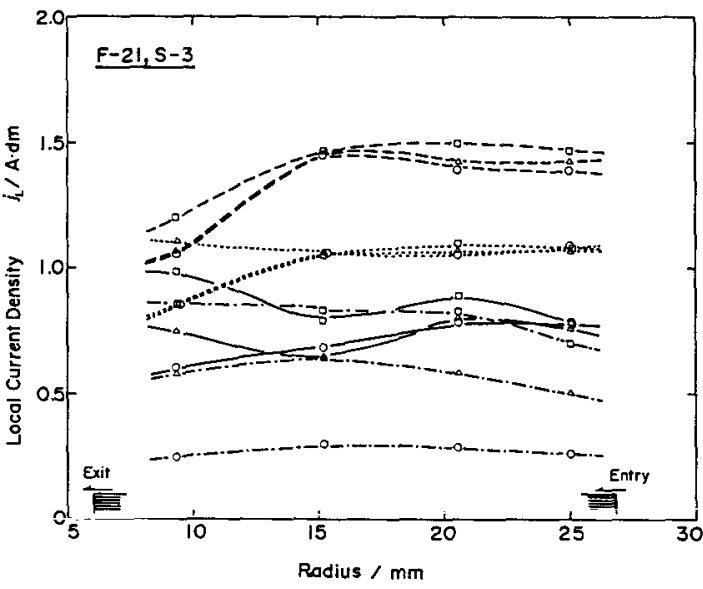

Fig. 8 Distribution of 1 imiting current density on $S-3$ in $F-21.1 \mathrm{~g}=3 \mathrm{~mm}$. Four fins with $\mathbf{f}_{\mathrm{h}}=2 \mathrm{~mm}$.

Rotationa1 speed ( $\mathrm{rpm}$ ):- $-0,-700, \cdots-1200$, - 2000. Volumetric flow rate $\left(\mathrm{cm}^{2} \cdot \mathrm{s}^{-1}\right)$ : $0-56, \Delta-278, \square-556$. 
With rotation, the results from the electrodes without fins show $(5,6)$ that mass transfer has a minimum in the intermediate part of the electrode surface, and at the inner and the outer part of the electrode surface it has very high values. This is explained by mass transfer in the outer part of the electrodes being dominated by the rotational motion, but the effect decreases towards the center as the tangential velocity decreases and the accelerating radial velocity component becomes more important in the inner part of the electrode.

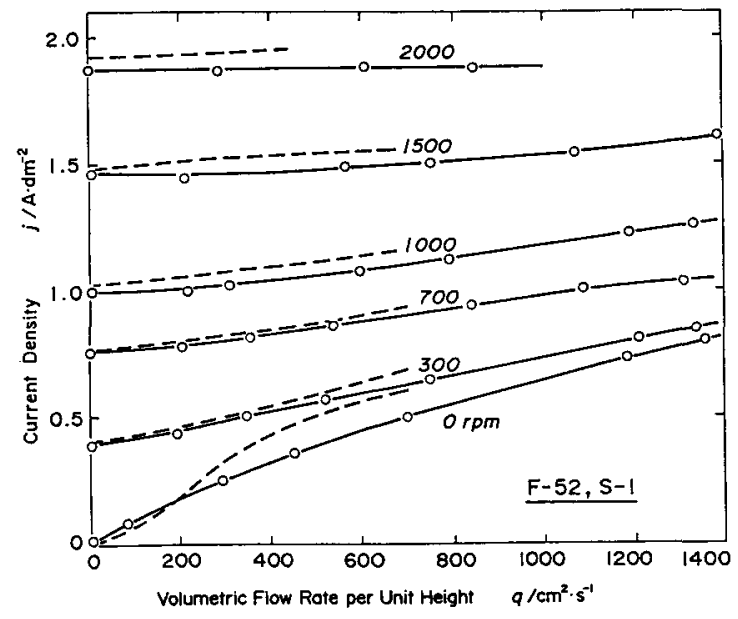

Fig. 9 Diffusion 1 imiting currents with different fin height on S-1 in F-52.

Solid 1ine: $1 \mathrm{~g}^{=} 3 \mathrm{~mm}, \mathrm{f}_{\mathrm{h}}=2 \mathrm{~mm}$; broken $1 \mathrm{ine}$ : $1_{\mathrm{g}}=6 \mathrm{~mm}, \mathrm{f}_{\mathrm{h}}=5 \mathrm{~mm}$.
In Figs. 7 and 8, at over 1200 rpm, the limiting currents are even or high in the intermediate part of the electrodes and are nowhere dominated by either the radial velocity or the tangential velocity.

The agitation by the fins raises the complexity of the flow and it cannot be explained in a two dimensional fie $1 d$ by a tangential and a radial velocity. This leads to difficulies in expressing the diffusion limiting current by a simple function of $q$ and $\omega$, (a product of $q$ and $w$ with constant exponents).(3) The fins flatten the limiting current across the electrode surface and this improves the properties of the cell.

Diffusion limiting currents for different heights of fins on the $S-1$ surface in F-52 are shown in Fig. 9, and for different numbers of fins on the S-1 and the S-3 in F-92 are shown in Figs. 10 and 11 .

Figure 9 shows that the limiting currents for the electrodes with 5 mmigh fins are just a little larger than for $2 \mathrm{~mm}$ fins. The differences are very small and a fin height of $2 \mathrm{~mm}$ or less is preferable, since a decrease in fin height leads to decreases in the energy consumption by decreasing cel1 voltage. This is important in electrolyzing dilute solutions without a supporting electrolyte

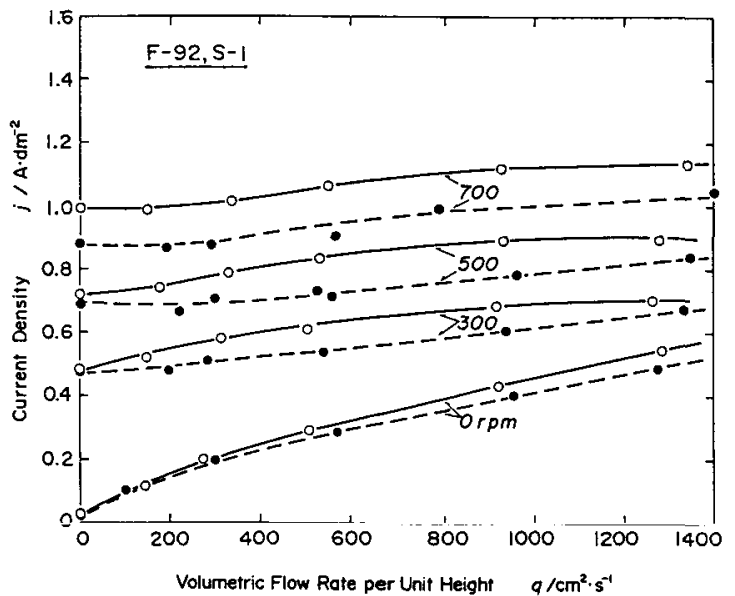

Fig. 10 Diffusion 1 imiting currents with different numbers of fins ( $f_{h}=2 \mathrm{~mm}, 1_{\mathrm{g}}=3$ inim) on $S-1$ in F-92.

Solid lines: four fins; broken lines: eight fins.

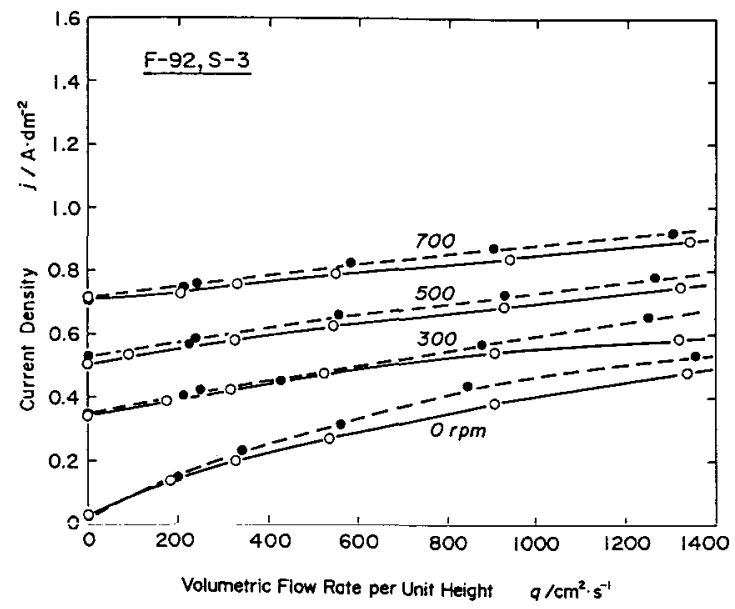

Fig. 11 Diffusion limiting currents with different numbers of fins $\left(\mathrm{f}_{\mathrm{h}}=2 \mathrm{~mm}, \mathrm{l}_{\mathrm{g}}=3\right.$ $\mathrm{mm}$ ) on $\mathrm{S}-3$ in $\mathrm{F}-92$.

Solid lines: four fins; broken lines: eight fins. 
like in the electrolytic recovery of metals and the electrochemical treatment of waste water.

In Figs. 10 and 11 the limiting currents for electrodes with eight fins are a 1ittle smaller than with four fins on the S-1 surface and a 1 ittle 1 arger on the S-3 surface but the differences are small.

\section{Conclusions}

The agitation of fins attached to electrodes flattens the distribution of the limiting current on the electrode surface and elevates the mass transfer rate.

A distance of $1 \mathrm{~mm}$ from the top of fins to the opposite surface is smallenough for the fins to increase the effective agitation of the opposite surface.

Varying the fin height from 2 to $5 \mathrm{~mm}$ and the number of fins from 4 to 8 has on $1 \mathrm{y}$ a sma11 effect on the 1 imiting currents.

\section{References}

1) T. Sasaki and T. Ishikawa, Electrochim. Acta, 31,745 (1986).

2) T. Sasaki, Y. Shinno, and T. Ishikawa, Bu11. Fac. Eng. Hokkaido Univ., 132 , $41(1986)$.

3) T. Sasaki and T. Ishikawa, DENKI KAGAKU, 55, 145 (1987).

4) M. Fleischmann, J. Ghoroghchian, and R. E. W. Jansson, J. Appl. Electrochem., 9, 437 (1979).

5) R. E. W. Jansson and R. J. Marsha11, Chem. Eng., 315, 769 (1976).

6) J. Ghoroghchian, R. E. W. Jansson, and R. J. Marsha11, Electrochim. Acta, 24, 1175 (1979). 